\title{
Studies on the Toxicological Effects of PFOA and PFOS on Rats Using Histological Observation and Chemical Analysis
}

\author{
Lin Cui · Qun-fang Zhou · Chun-yang Liao · \\ Jian-jie Fu $\cdot$ Gui-bin Jiang
}

Received: 8 April 2008/ Accepted: 23 June 2008/Published online: 26 July 2008

(C) Springer Science+Business Media, LLC 2008

\begin{abstract}
As an emerging class of environmentally persistent and bioaccumulative contaminants, perfluorinated compounds (PFCs), especially perfluorooctanoic acid (PFOA) and perfluorooctane sulfonate (PFOS), have been ubiquitously found in the environment. Increasing evidence shows that the accumulated levels of PFCs in animals and the human body might cause potential impairment to their health. In the present study, toxicological effects of PFOA and PFOS on male Sprague-Dawley rats were examined after 28 days of subchronic exposure. Abnormal behavior and sharp weight loss were observed in the high-dose PFOS group. Marked hepatomegaly, renal hypertrophy, and orchioncus in treated groups were in accordance with the viscera-somatic indexes of the liver, kidney, and gonad. Histopathological observation showed that relatively serious damage occurred in the liver and lung, mainly including hepatocytic hypertrophy and cytoplasmic vacuolation in the livers and congestion and thickened epithelial walls in the lungs. PFOA concentrations in main target organs were in the order of kidney $>$ liver $>$ lung $>$ (heart, whole blood) $>$ testicle $>$ (spleen, brain), whereas the bioaccumulation order for PFOS was liver $>$ heart $>$ kidney $>$ (whole blood) $>$ lung $>$ (testicle, spleen, brain). The highest concentration of PFOA detected in the kidney exposed to $5 \mathrm{mg} / \mathrm{kg} /$ day was $228 \pm 37 \mu \mathrm{g} / \mathrm{g}$ and PFOS in the liver exposed to $20 \mathrm{mg} / \mathrm{kg} /$ day reached the highest level of $648 \pm 17 \mu \mathrm{g} / \mathrm{g}$, indicating that the liver, lung, and kidney might serve as the main target organs for PFCs. Furthermore, a dose-dependent accumulation of PFOS in various tissues
\end{abstract}

L. Cui · Q.-f. Zhou · C.-y. Liao · J.-j. Fu · G.-b. Jiang ( $₫)$ State Key Laboratory of Environmental Chemistry and Ecotoxicology, Research Center for Eco-Environmental Sciences, Chinese Academy of Sciences, Beijing 100085, China e-mail: gbjiang@rcees.ac.cn was found. The accumulation levels of PFOS were universally higher than PFOA, which might explain the relative high toxicity of PFOS. The definite toxicity and high accumulation of the tested PFCs might pose a great threat to biota and human beings due to their widespread application in various fields.

\section{Introduction}

Perfluorinated compounds (PFCs) have been widely used in industrial application and civilian production because of their high stability and strong lipophobic and hydrophobic properties (Giesy and Kannan 2002; Key et al. 1997). Perfluorooctanic acid (PFOA) and perfluorooctane sulfonate (PFOS) are two of the most principal representatives. The former is one of the final products of PFCs by metabolism in an organism and the environment, and the latter is an important perfluorosurfactant and a precursor of many other homologues of PFCs. As emerging pollutants, PFCs can enter into water, atmosphere, soil, sludge, and other environmental media by all means, and they have caused widespread pollution. They can be transferred, bioaccumulated, and biomagnified along food chains and their presence has been found in various tissues of many wildlife species and even in the human body (Giesy and Kannan 2001; Houde et al. 2006; Kannan et al. 2004). Due to their long persistence, bioaccumulation, and potential toxicity to organisms (Giesy and Kannan 2001), United Nations Environment Programme (UNEP) has considered adopting PFOS and 96 PFOS-related substances as candidates of persistent organic pollutants (POPs) in the Stockholm Convention in November 2006. Considerable public attention has been paid to the toxicities of PFCs 
because the available toxicological data still greatly limited the risk assessment of the environmental pollution caused by PFCs.

Previous research concerned with hematology, urinalysis, and enzymic assay was performed to indicate the toxicities of PFCs by short-term oral administration at high doses (Dean et al. 1978; Ylinen et al. 1990). Cell morphology observations were also carried out to reflect biochemical effects induced by PFCs. The increase of the total cytochrome P-450 (CYP450) content, benzphetamine $N$-demethylase activity (Pastoor et al. 1987), and carboxylesterase activity (Hosokawa and Satoh 1993) were found along with the hyperplasia of the smooth endoplasmic reticulum in the exposed rat liver (Pastoor et al. 1987). PFCs were found to increase the numbers and size of peroxisomes, induce peroxisomal fatty acid $\beta$-oxidation, accelerate CYP450-mediated $\omega$-hydroxylation of lauric acid, and bring about the hypolipemic effect (Berthiaume and Wallace 2002; Haughom and Spydevold 1992; Ikeda et al. 1985). The neurotoxicity, genotoxicity, and reproductive and developmental toxicity have evoked much concern. PFOS has been testified to affect the neuroendocrine system in rats as an endocrine disruptor (Austin et al. 2003). Although the genotoxicity of PFCs has not been revealed clearly, alterations in expression of some specific genes in rats due to PFCs exposure have been identified by microarray techniques. Genes significantly induced by PFOS treatment were primarily those for fatty-acid-metabolizing enzymes, CYP450, or those involved in hormone regulation (Hu et al. 2005). Comparatively, suppression of genes expression caused by PFOA administration was largely related to the transport of lipids, inflammation and immunity, and especially cell adhesion (Guruge et al. 2007). A multigenerational reproduction study by $3 \mathrm{M}$ Company indicated that PFOS induced a marked reduction of body and perinatal viability in rats but had no adverse effect on mating and fertility (Butenhoff et al. 2002). Two developmental studies demonstrated that high-dose exposure of PFOS during pregnancy could lead to both maternal and developmental toxicity in the rat and mouse (Lau et al. 2003; Thibodeaux et al. 2003). Postnatal survival of neonatal rats and mice was seriously compromised. In addition, the growth and development accompanied by hypothyroxinemia in the surviving rat pups were delayed. Distribution and accumulation patterns of PFCs in exposed experimental animals such as rats, mice, and hamsters were investigated and high levels of PFCs were found in the liver and blood (or serum) (Dupont Haskell Laboratory 1982; Ylinen et al. 1990). Some toxicological profiles of PFCs can be obtained based on the previous studies; however, little information on the histological alterations and accumulation patterns has been compared for different PFCs, such as PFOA and PFOS, at different exposure levels.
In this article, histopathological alterations were carefully investigated in various organs in the Sprague-Dawley (SD) rats exposed to PFOA and PFOS at 5 and $20 \mathrm{mg} / \mathrm{kg} /$ day. The distribution of two perfluorochemicals in the rats was compared for the evaluation on histopathological effects for both tested compounds, which might offer the best understanding of the toxicological effects induced by PFCs.

\section{Materials and Methods}

\section{Reagents}

Pentadecafluorooctanoic acid (PFOA, 96\%) and perfluorooctane sulfonate potassium (PFOS, 98\%) were purchased from Sigma-Aldrich (St. Louis, MO). Tetrabutylammonium (TBA, 97\%) hydrogen sulfate and methyl tert-butyl ether (MTBE, 99\%) were purchased from Alfa Aesar (Ward Hill, MA) and Acros (Morris Plains, NJ), respectively. The $0.5 \mathrm{M}$ TBA solution (adjusted to $\mathrm{pH} 10.0$ with sodium hydroxide) and the $0.25 \mathrm{M}$ sodium carbonate buffer solution $\left(\mathrm{Na}_{2} \mathrm{CO}_{3} /\right.$ $\mathrm{NaHCO}_{3}$ ) were prepared with Milli-Q water (Millipore, Bedford, MA). Methanol was high-performance liquid chromatography (HPLC) grade from J. T. Baker Inc. (Phillipsburg, NJ). Sodium perfluoro-1-[1,2,3,4- $\left.{ }^{13} \mathrm{C}_{4}\right]$ octanesulfonate (MPFOS, ${ }^{13} \mathrm{C}_{4}{ }^{12} \mathrm{C}_{4} \mathrm{~F}_{17} \mathrm{SO}_{3} \mathrm{Na}$, chemical purity $\geq 98 \%$, isotopic purity $\geq 99 \%$ ), as the internal standard, was purchased from Wellington Laboratories (Ontario, Canada) and the work solution at the level of $100 \mathrm{ng} / \mathrm{mL}$ was prepared in methanol. All solutions described here were stored at $4^{\circ} \mathrm{C}$ and adjusted to room temperature just before use.

The oral administration solutions of PFOA and PFOS were prepared daily by diluting suitable amounts of the chemicals in Milli-Q water. The concentrations of PFOS and PFOA $(0.5$ and $2 \mathrm{mg} / \mathrm{mL})$ were verified by the analysis using a HPLC-electrospray tandem mass spectrometry system (HPLC/ESI-MS/MS, ABI 3200 triple quadruple mass spectrometer configured with ESI; ABI, USA), equipped with an automatic sample injector (AS50; Dionex, USA), as described previously by Zhang et al. (2007).

Animals

The use and care of animals followed the guideline of The Animal Care \& Welfare Committee at the Institute of Materia Medica, Chinese Academy of Medical Sciences and Peking Union Medical College (CAMS \& PUMC). Fifty male SD rats (specific pathogen-free grade, around 2 months old, 190-210 g) were purchased from Beijing WeiTong Lihua Experiment Animal Technology Ltd. (Beijing, China) and were housed individually in stainless- 
steel wire-mesh cages. Certified laboratory chow and sterilized water were available ad libitum over the experiment. Animal rooms were targeted at a temperature of $22-24^{\circ} \mathrm{C}$ and a relative humidity of $40-60 \%$ and were artificially illuminated with a 12-h light/dark cycle. Prior to exposure, all rats were acclimatized in the experimental environment for a 1-week quarantine period.

\section{Exposure Protocol}

The acclimatized rats were randomized into 5 groups (10 per group) and administrated with the PFC solutions by gavage (i.e., intragastric intubation) at a standard volume of $10 \mathrm{~mL} / \mathrm{kg}$ of body weight individually obtained prior to administration. The first group (G0) was used as the blank for the control free from PFCs, which received Milli-Q water only. The other four groups were administered $5 \mathrm{mg} /$ $\mathrm{kg} /$ day of PFOA (named by G1), $20 \mathrm{mg} / \mathrm{kg} /$ day of PFOA (G2), $5 \mathrm{mg} / \mathrm{kg} / \mathrm{day}$ of PFOS (G3), and $20 \mathrm{mg} / \mathrm{kg} / \mathrm{day}$ of PFOS (G4), respectively. The gavage was preformed once a day. The whole exposure duration lasted 28 days. All of the rats were carefully observed for their activities and the body weights were measured every week. All of the rats were sacrificed after the exposure. Approximately $4 \mathrm{~mL}$ of blood were obtained from femoral arteries and the tissue samples, including the liver, kidney, lung, heart, spleen, testis, and brain, were collected and weighed. A cubical sample of about $0.5 \mathrm{~cm}^{3}$ was removed from each tissue and immediately immersed into a formaldehyde solution $(10 \%$, $\mathrm{v} / \mathrm{v}$ ) for histopathological observation. The remaining tissues and blood samples were stored at $-40^{\circ} \mathrm{C}$ for chemical analysis.

\section{Histopathological Pretreatment}

The histological specimen was prepared briefly as follows. Each fixed tissue sample was dehydrated with gradient solutions of ethanol $(70 \%, 80 \%, 85 \%, 90 \%$, and $95 \%)$, which was subsequently substituted by dimethylbenzene. After being embedded into paraffin at $58-60^{\circ} \mathrm{C}$, the tissue specimen was sliced up by an automatic microtome and then dyed by hematoxylin and eosin (H\&E) staining. The prepared histological sections from the tissues in the control and exposure groups (more than five samples per tissue per group) were observed with an optical microscope (OLYMPUS BX41, Japan).

Analytical Procedure of PFOA and PFOS in Tissue Samples

Sample pretreatments for the analysis of PFOA and PFOS in tissue samples were in accordance to previous references with some minor modifications (Hansen et al. 2001). In short, the whole-blood samples and tissue homogenates were diluted with Milli-Q water in a suitable proportion. A portion of homogenate dilution $(1 \mathrm{~mL})$, spiked with $50 \mu \mathrm{L}(100 \mathrm{ng} / \mathrm{mL})$ MPFOS as an internal standard, was mixed thoroughly with $1 \mathrm{~mL}$ of $0.5 \mathrm{M}$ TBA (pH 10.0) and $2 \mathrm{~mL}$ of $0.25 \mathrm{M}$ sodium carbonate buffer in a $15-\mathrm{mL}$ polypropylene (PP) tube. After the addition of $5 \mathrm{~mL}$ of MTBE, the mixture was shaken at $250 \mathrm{rpm}$ for $20 \mathrm{~min}$ and then the organic and aqueous layers were separated by centrifugation at $3000 \mathrm{rpm}$ for $15 \mathrm{~min}$. About $5 \mathrm{~mL}$ of organic aliquot (MTBE) was removed and the residual mixture was rinsed again twice with MTBE for extraction and separation. The organic extracts were combined in a second PP tube, evaporated under nitrogen stream, resuspended in $0.5 \mathrm{~mL}$ methanol for solvent substitution, and extracted on a SPE column (Oasis HLB $200 \mathrm{mg}, 6 \mathrm{cc}$, Waters, USA), which was subsequently preconditioned with $5 \mathrm{~mL}$ of methanol and $5 \mathrm{~mL}$ of water. After washing with $5 \mathrm{~mL}$ of $40 \%$ methanol in water (v/v), the column was vacuumed as dry as possible. The elution ran through the column at a flow rate at $1 \mathrm{drop} / \mathrm{s}$ under vacuum suction and was discarded. After that, the column was extracted with $10 \mathrm{~mL}$ of methanol without vacuum and the eluate was concentrated to $1 \mathrm{~mL}$ again and stored at $4^{\circ} \mathrm{C}$ until analysis.

Separation and quantitation of PFOA and PFOS in the final extract were performed by HPLC/ESI-MS/MS. An aliquot of $10 \mu \mathrm{L}$ was injected into a guard column connected sequentially to an analytical column (Capcell Pak UG120 $\mathrm{C}_{18}$ column, $3-\mu \mathrm{m}$ particle diameter, $50 \mathrm{~mm} \times 2.0 \mathrm{~mm}$ inner diameter; SHIDSEIDO, Japan) with methanol (A) and $2 \mathrm{mM}$ ammonium acetate (B) as the mobile phase $(0.1 \mathrm{~mL} / \mathrm{min})$. The percentage of methanol varied during the chromatographic run to give the following values at the specified times: $30 \% \mathrm{~A}$ at $0-0.1 \mathrm{~min}$, linear gradient from $30 \%$ to $100 \%$ A in 10 min, keep at $100 \% \mathrm{~A}$ for $10-18 \mathrm{~min}$, and then linear gradient from $100 \%$ to $30 \% \mathrm{~A}$ in $0.1 \mathrm{~min}$, keep at $30 \% \mathrm{~A}$ for $18.1-$ $25 \mathrm{~min}$. The mass detector was performed in the "ESI (-) mode" and the key parameters were set as follows: temperature, $400^{\circ} \mathrm{C}$; curtain gas, $15 \mathrm{psi}$; collision gas, $3 \mathrm{psi}$; ion source gas 1, $55 \mathrm{psi}$; ion source gas 2, $45 \mathrm{psi}$; ion spray voltage, $-4500 \mathrm{~V}$. MPFOS was used to quantify, and monitored ion pair transitions (parent ion $\rightarrow$ daughter ion) for confirmation of PFOA, PFOS, and MPFOS were $412.8 \rightarrow 369.0,498.8 \rightarrow 79.9$, and $502.9 \rightarrow 80.0$ separately. Dosing preparations were testified to be at the targeted concentrations, homogeneous, and stable, and no PFOA or PFOS was detected in Milli-Q water.

The feasibility of the method was certified with the recoveries in the range of $75.2-109.6 \%$ and $79.0-95.4 \%$ for PFOA and PFOS, respectively. 
Statistics

All data were expressed as mean \pm sd. One-way analysis of variance (ANOVA) was used to pairwise compare differences between the treated and control groups. $p<0.05$ was regarded as a statistically significant difference.

\section{Results and Discussion}

\section{Behavior Observations}

During the entire exposure period, all rats were carefully observed for their activities, ingestion, excretion, psychosis, mortality, and moribundity. The rats in G0 showed normal activities during the experimental procedure without death. Similar phenomena were observed in both G1 and G3 groups during the first 2 weeks, whereas slight abnormalities were found from the third week, including reduced activity, decreasing food uptake, cachexia, and lethargy. All of the rats in G2 showed relatively more sensitivity to external stimuli, in addition to the ethological abnormity observed above. Obvious pathological alterations were observed in rats dosed at $20 \mathrm{mg} / \mathrm{kg} /$ day PFOS. All diseased behaviors described earlier were found from the very beginning of the exposure. Death occurred after 11 days of exposure and all rats in G4 finally died within 26 days. At necropsy, typical symptoms such as bleeding around eye sockets and snoots, and tumescence and yellow stain at the urogenital region were observed in the dead rats (Fig. 1). PFOS exhibited relatively higher toxicity than PFOA based on these ethological hints and mortality occurrence.

Weekly body weight monitoring showed an increase trend of the average body weight in G0 over the study period, as shown in Fig. 2. Similar changes were observed in both G1 and G3, with a slightly faster increase in the second week and a slightly slower increase in the last 2 weeks. Reduced activities first and anorexia later might explain the change trends of body weights in G1 and G3. Although a slight increase in body weight was observed in G2 during the exposure period, the weight gains were

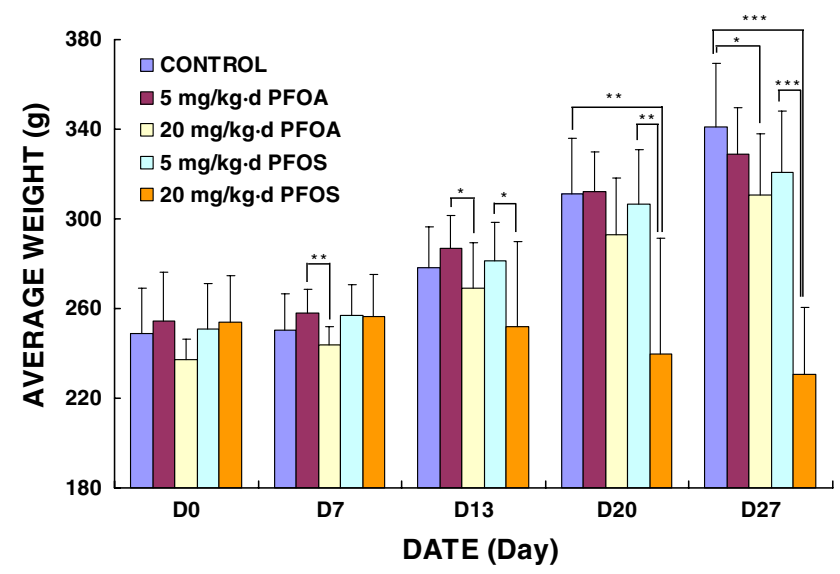

Fig. 2 The changes in the rat body weights during 28 days of exposure. Data are expressed as mean $\pm \mathrm{sd}(n=10)$. $* p<0.05$, $* * p<0.01$, *** $p<0.001$, as compared with control group or compared between two groups indicated, one-way ANOVA

significantly lower than those in the control group and lowdose exposure group (G1) $(p<0.05)$, which showed that PFOA exposure induced dose-related inhibition in the body weight gains of the rats. For the rats in G4, except for the first exposure week, the sharp loss of body weights was evident to show the high toxicity of PFOS at the given exposure level. Food consumption in every group was recorded daily (data not shown) throughout the exposure. Mean food consumption in G1, G2, and G3 were slightly reduced in a similar manner compared to the control (G0), indicating that both toxicants have no apparent influence on the food consumption in these groups. However, the significant decrease of food consumption was observed in G4, which was responsible for the reduction in body weights of the rats exposed to a high dose of PFOS.

\section{Viscera Index}

The viscera indexes, including the hepatosomatic index (HSI), renal-somatic index (RSI) and gonad-somatic index (GSI) calculated as formula 1 was used to evaluate hyperplasia, swelling, or atrophy of the organs induced by PFCs exposure. As shown in Fig. 3, the HSI, RSI, and GSI in the exposure groups were all significantly higher than
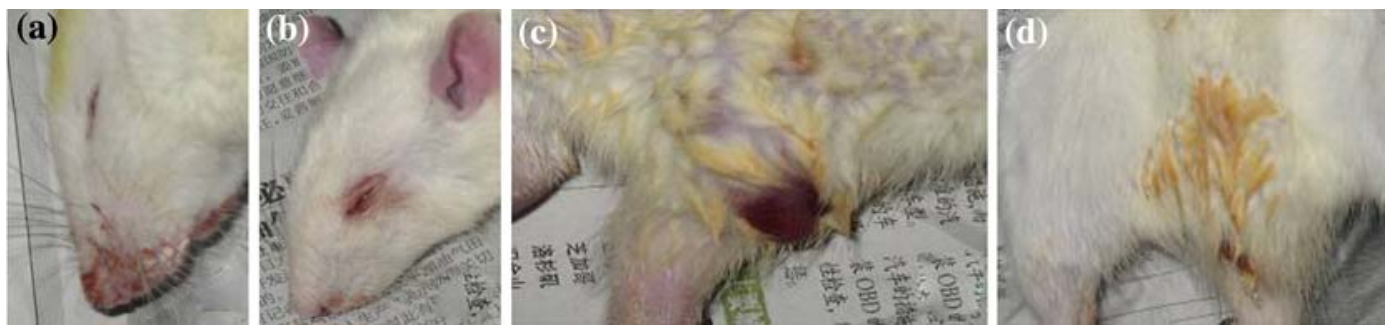

Fig. 1 Typical symptoms, including bleeding around snoots (a) and eye sockets (b) and tumescence (c) and yellow stain (d) at urogenital region, were observed in all of the dead rats $(n=10)$ dosed at $20 \mathrm{mg} / \mathrm{kg} /$ day of PFOS 


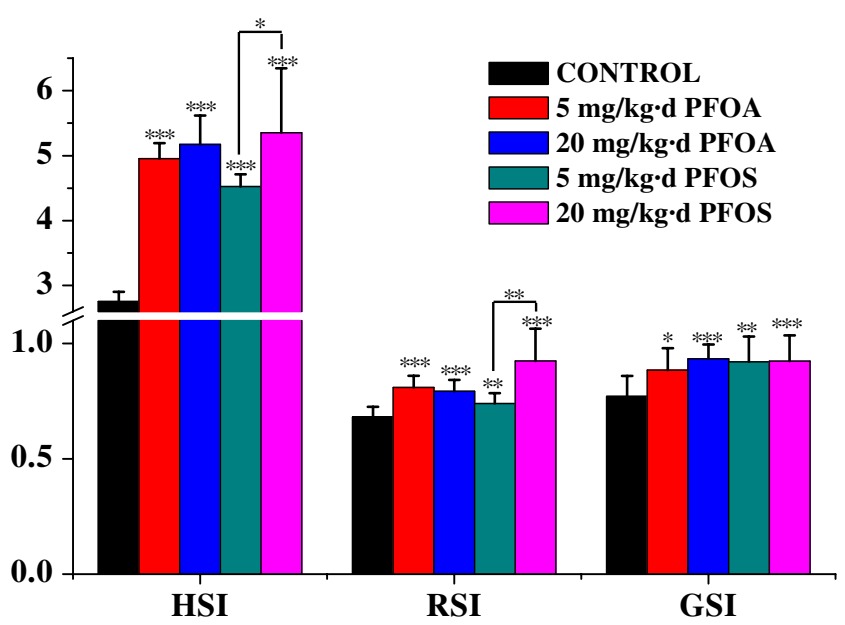

Fig. 3 The changes in the viscera indexes of the control and exposure groups after PFC treatment. HSI, RSI, and GSI stand for hepatosomatic index, renal-somatic index, and gonad-somatic index, respectively. Data are expressed as mean \pm sd $(n=10)$. $* p<0.05$, ** $p<0.01$, *** $p<0.001$, as compared with control group or compared between two groups indicated, one-way ANOVA

those of the control $(p<0.05)$, indicating that obvious hepatomegaly, renal hypertrophy, and orchioncus could be caused by PFOA and PFOS. The increases of HSI and RSI in the PFOS groups showed a dose dependence $(p<0.05)$.

$$
\begin{aligned}
& \text { Viscera Index }(\text { HSI/RSI/GSI }) \\
& =\frac{\text { Visceral }(\text { Hepatic } / \text { Renal } / \text { Gonad }) \text { Weight }(\mathrm{g})}{\text { Rat Weight }(\mathrm{g})} \times 100 \text {. }
\end{aligned}
$$

Histopathological Observations

\section{Liver}

At necropsy, the liver tissues from the control rats showed normal red and even texture as displayed in Fig. 4a. No
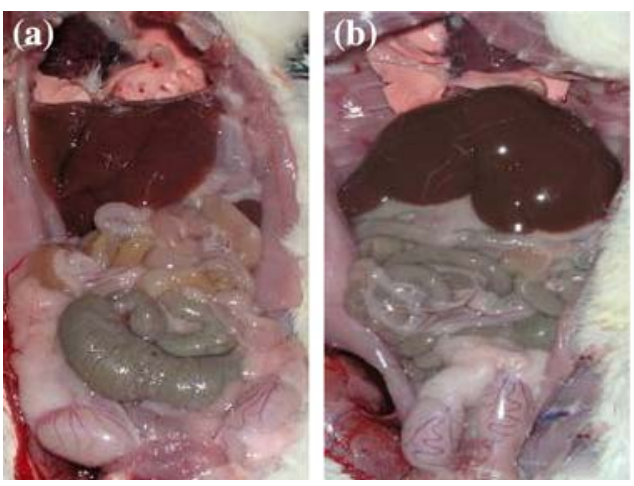

Fig. 4 Observable symptoms in rat livers exposed to $20 \mathrm{mg} / \mathrm{kg} / \mathrm{day}$ of PFOA or PFOS. All of the rats $(n=10)$ in the control and four exposure groups were checked. Compared to the normal liver tissues from the control (a), no distinguishable optical symptoms were found in $5-\mathrm{mg} / \mathrm{kg} /$ day PFOA and PFOS dose groups (images not shown); distinguishable optical symptoms were found in the lowdose exposure groups. The liver tissues from G3 exhibited slight tumefaction and dark color (Fig. 4b), whereas obvious tumefaction and discoloration symptoms were universal in the livers from G4 (Fig. 4c). Some yellow stains on the hepatic surface were remarkable in G4 (Fig. 4d), showing that abnormality related with hepatic inflammation could be induced by PFOS.

Based on the microscope observation, normal hepatic sinusoid and hepatic lobules can be found clearly in G0 (Fig. 5a). Cytoplasmic vacuolation (Fig. 5b, e), focal or flakelike necrosis (Fig. 5d, e), and hepatocellular hypertrophy (Fig. 5e) were universally observed in each treated group. Fatty degeneration (Fig. 5b), angiectasis and congestion in the hepatic sinusoid or central vein (Fig. 5b), and acidophil lesion (Fig. 5c) were induced by PFOA exposure (G2), whereas much more serious symptoms appeared in PFOS exposure groups, especially for G4. The dose-related hepatic toxicity included focal hemorrhage (Fig. 5d), erythrocytic transudation (Fig. 5d), and focal hepatocytic degeneration accompanying by inflammatory cellular infiltration (Fig. 5e).

Previous studies have identified the liver as the primary target organ for both acute and chronic exposure to PFCs. In the study by Seacat et al. (2003), male rats were administrated the highest dose $(0.002 \%)$ of PFOS in the diet for 14 weeks, achieving a cumulative dose of $\sim 130 \mathrm{mg} / \mathrm{kg}$, which was close to that $(140 \mathrm{mg} / \mathrm{kg})$ in our study following 4 weeks of $5 \mathrm{mg} / \mathrm{kg} /$ day PFOS dosing. PFOA and PFOS were also considered as potent peroxisome proliferators in rats, mice, and rhesus (Abdellatif and Preat 1990, 1999; Dzhekova et al. 2001; Panaretakis et al. 2001), and the peroxisome proliferation induced by both chemicals especially at a high dose caused the formation of hepatocellular hypertrophy (Haughom and Spydevold 1992; Kennedy et al. 2004). The increase in liver mass was commonly observed in this study, which, in part, is
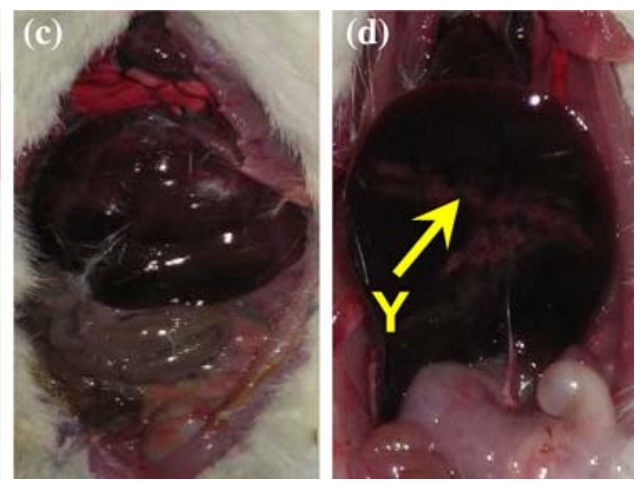

however, tumefaction and dark color were observed in the livers from the $20-\mathrm{mg} / \mathrm{kg} /$ day PFOA dose group (b) and obvious tumefaction, discoloration, and yellow stains (Y) on the liver surface from the 20$\mathrm{mg} / \mathrm{kg} /$ day PFOS dose group $(\mathbf{c}, \mathbf{d})$ 
Fig. 5 Histopathological sections of rat liver from the control and exposure groups. $\mathrm{H} \& \mathrm{E} ;(\mathbf{a}-\mathbf{d}), \times 200$;

(e), $\times$ 400. (a) control; (b, c) $20 \mathrm{mg} / \mathrm{kg} /$ day of PFOA; (d, e) $20 \mathrm{mg} / \mathrm{kg} / \mathrm{day}$ of PFOS. In Figs. 5-8, the histological sections of every tissue from more than five rats per group were checked. The images with slight damages from the 5-mg/ $\mathrm{kg}$ /day exposure groups were not shown. Compared to normal hepatic sinusoid and clear hepatic lobules in the control (a), various degrees of histopathological changes were observed in all exposure groups, especially in the $20-\mathrm{mg} / \mathrm{kg} /$ day exposure groups (b-e), mainly including fatty degeneration $(\mathrm{Fd})$, angiectasis in central vein, congestion (C) in hepatic sinusoid, hepatocytic acidophil lesion (Al), focal hemorrhage $(\mathrm{H})$ induced by erythrocytic transudation, flakelike or focal hepatocytic necrosis $(\mathrm{N})$, focal hydropic degeneration [i.e., cytoplasmic vacuolation (V)], hepatocytic hypertrophy $(\mathrm{Ht})$, and inflammatory cellular infiltration (I)

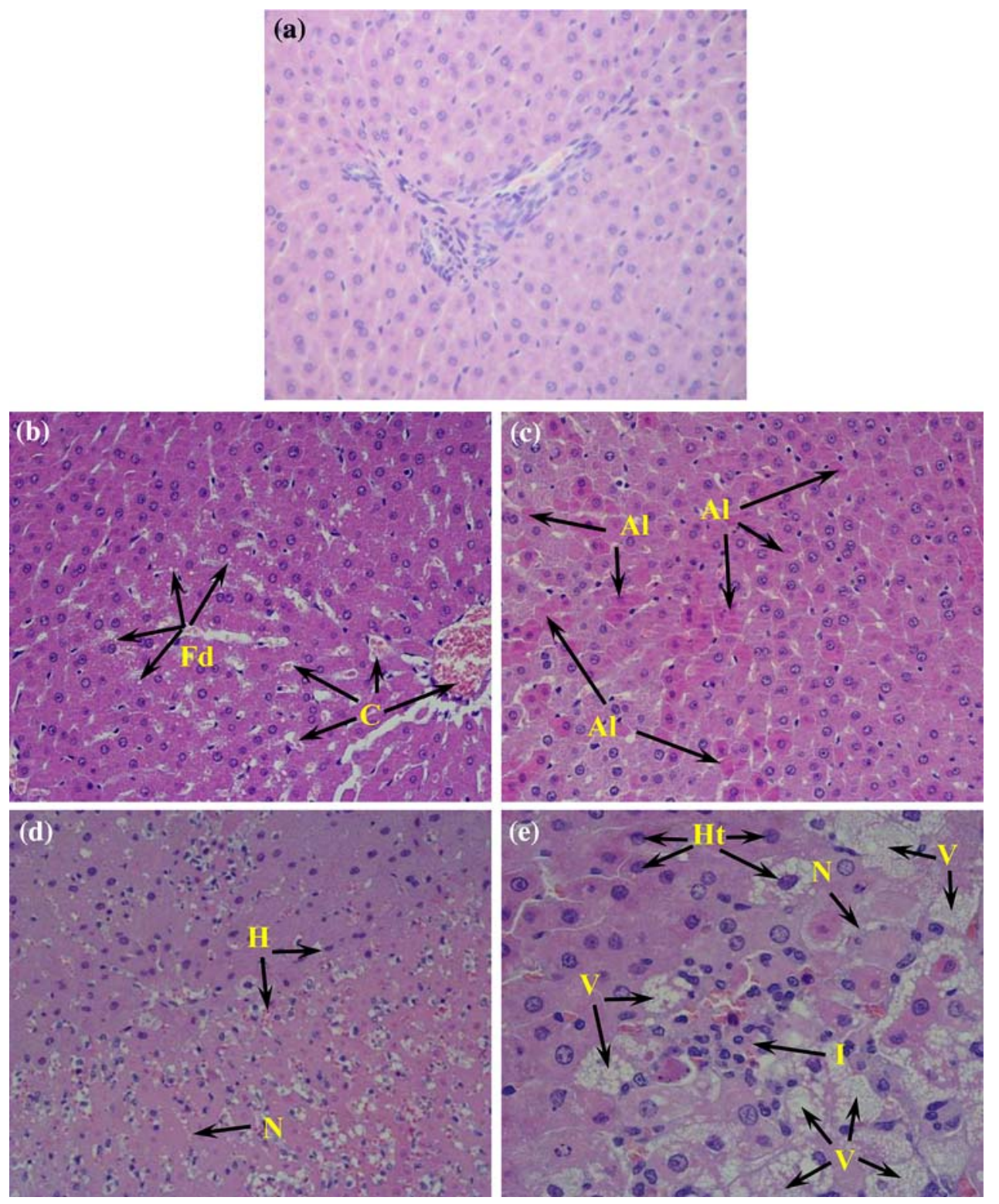

attributed to proliferation of peroxisome, smooth endoplasmic reticulum, and mitochondria (Berthiaume and Wallace 2002; Pastoor et al. 1987), but not hepatocellular hyperplasia (no increase in nuclear DNA). Although the toxicity mechanism of PFCs is still not clear, it is commonly accepted that PFCs could result in the induction of hepatic catalase, acyl-CoA oxidase, and glutathione by altering cytosolic enzymes activities, such as glutathionerelated enzymes in rodent liver (Chen et al. 2001; DePierre 2002; O'Brien et al. 2001). The potential hepatic toxicity was confirmed by histopathological observation herein.

\section{Lung}

The lung is another important target organ in addition to the liver in rats (Dean et al. 1978; Grasty et al. 2003). In contrast to the structures of pulmonary alveolus and arteriole in G0 (Fig. 6a), pulmonary congestion (Fig. 6c, d) as well as focal or diffuse thickened epithelial walls (Fig. 6c, $\mathrm{d}$, and e) was commonly observed in the lungs at a low dose of chemical exposure (G1 and G3), whereas these pathological phenomenon became more obvious when the rats were treated with a high dose of PFOA (G2) or PFOS (G4). Focal or diffuse (neutrophil, acidophilia, and lymphocyte) cellular infiltration (Fig. 6c, e) and vasodilatation (Fig. 6c, d) also existed, specially notable in G4, due to leakage of erythrocytes (Figs. 6b, e). Comparatively, the most serious histopathological changes in lungs found in G4 might be related to labored breathing and bloodstain around snoots in the rats in this group. It was hypothesized that insufficient production of pulmonary surfactant by the alveolar type II cells, which was validated leading to 
Fig. 6 Histopathological sections of rat lung from the control and exposure groups. $\mathrm{H} \& \mathrm{E}, \times 200$. (a) control; (b, c) $20 \mathrm{mg} / \mathrm{kg} /$ day of PFOA; (d, e) $20 \mathrm{mg} / \mathrm{kg} /$ day of PFOS. The control (a) exhibited a normal structure of pulmonary alveolus and arteriole. Pathological changes in different degrees, such as focal hemorrhage $(\mathrm{H})$ induced by leakage of erythrocytes in pulmonary alveolus intracavity and bronchus cavities, focal or diffuse thickened epithelial walls $(\mathrm{T})$ of pulmonary alveolus, arteriostenosis (As) induced by thickened dissepiments, focal infiltration (I) of lymphocyte and neutrophil in stroma, vasodilatation $(\mathrm{Vd})$ and congestion with neutrophil aggregation in lumens, acidophilia infiltration (Ai) of granulocytes around arterioles and fibrosis (F), could be observed clearly in exposure groups, especially in the $20-\mathrm{mg}$ / $\mathrm{kg} /$ day exposure groups (b-e)
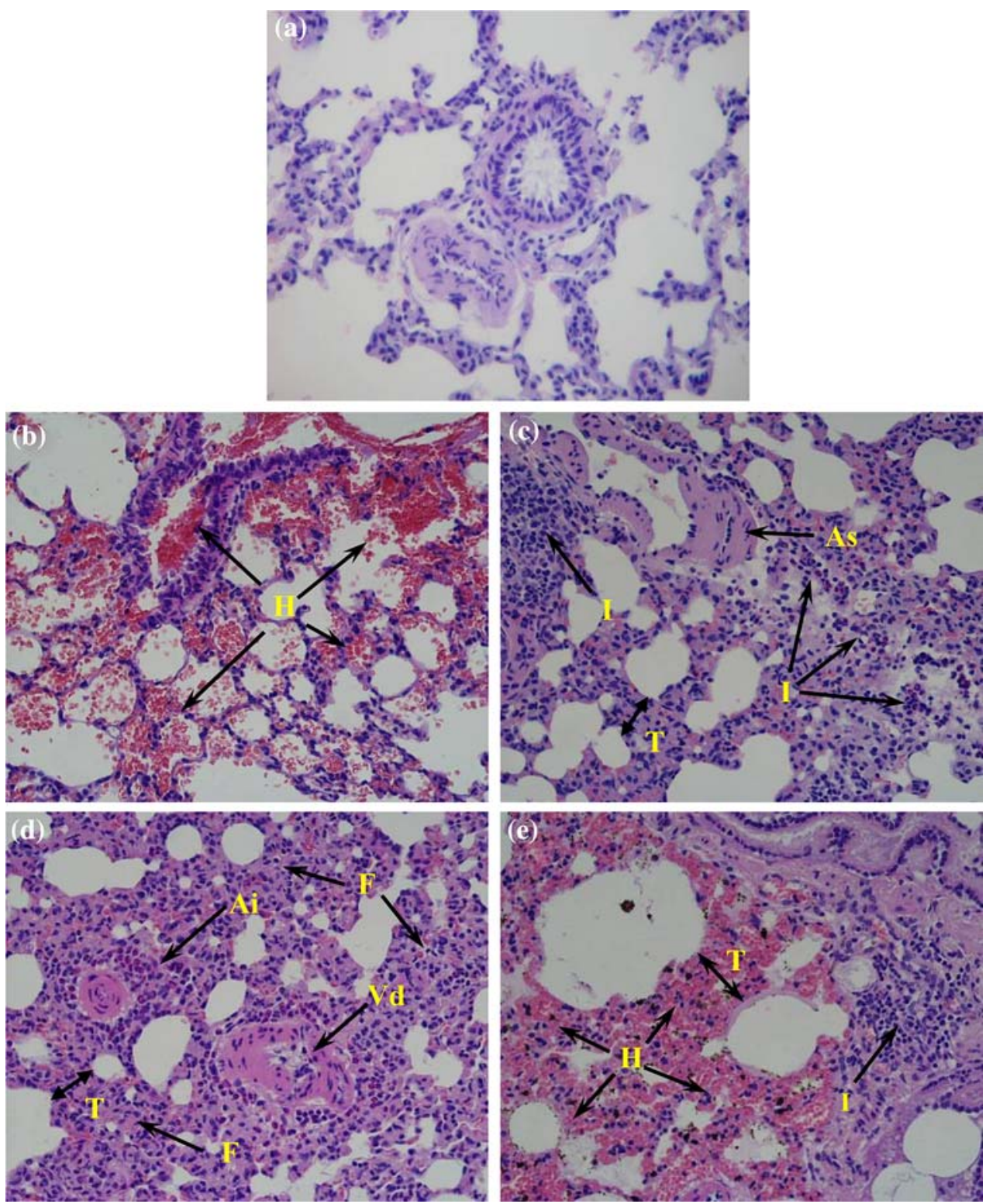

neonatal death in humans due to respiratory distress syndrome (Batenburg 1992; Creuwels et al. 1997; Hallman et al. 2001), was the major cause of death and severe pulmonary injury in rats. Exposure to PFCs has probably altered or inhibited the surfactant biosynthetic pathway in which PFCs influence some essential enzyme during the course of phospholipids synthesis (Fisher and Dodia 2001).

\section{Kidney}

It was found that some morphological and biochemical cases induced by PFCs occurred not only in the liver but also sometimes in the kidney (Kennedy et al. 2004). As an important organ in the metabolism, the potential histopathological effects on the kidney were also investigated. Compared to the normal renal corpuscle, renal cortex labyrinth, and medullary ray in the kidney from G0 (Fig. 7a, b), no significant differences were observed in the low-dose exposure groups (G1 and G3). Turbidness and tumefaction in the epithelia of the proximal convoluted tubule (Fig. 7c, e) were commonly observed in the highdose exposure groups (G2 and G4), accompanied by some mild symptoms, including congestion in the renal cortex and medulla (Fig. 7e, f) and enhanced cytoplasmic acidophilia (Fig. 7d). These symptoms indicated that the disturbances in the metabolism might be induced in the exposed rats.

\section{Other Organs}

Other important organs, including the spleen, brain, and testicle, were also studied. Slight congestion accompanying 
Fig. 7 Histopathological sections of rat kidney from the control and exposure groups.

$\mathrm{H} \& \mathrm{E}, \times 200$. (a, b) control; (c, d) $20 \mathrm{mg} / \mathrm{kg} /$ day of PFOA; (e, f) $20 \mathrm{mg} / \mathrm{kg} / \mathrm{day}$ of PFOS.

Compared to distinct structures of renal corpuscle $(\mathrm{Rc})$, renal cortex labyrinth (Rl), and medullary ray $(\mathrm{M})$ in the control $(\mathbf{a}, \mathbf{b})$, various degrees of histopathological changes appeared in exposure groups, especially in the $20-\mathrm{mg} / \mathrm{kg} / \mathrm{day}$ exposure groups (c-f), which mainly included turbidness and tumefaction in the epithelia of proximal convoluted tubule, enhanced acidophilia (A) in cytoplasm, and congestion (C) in renal cortex and medulla
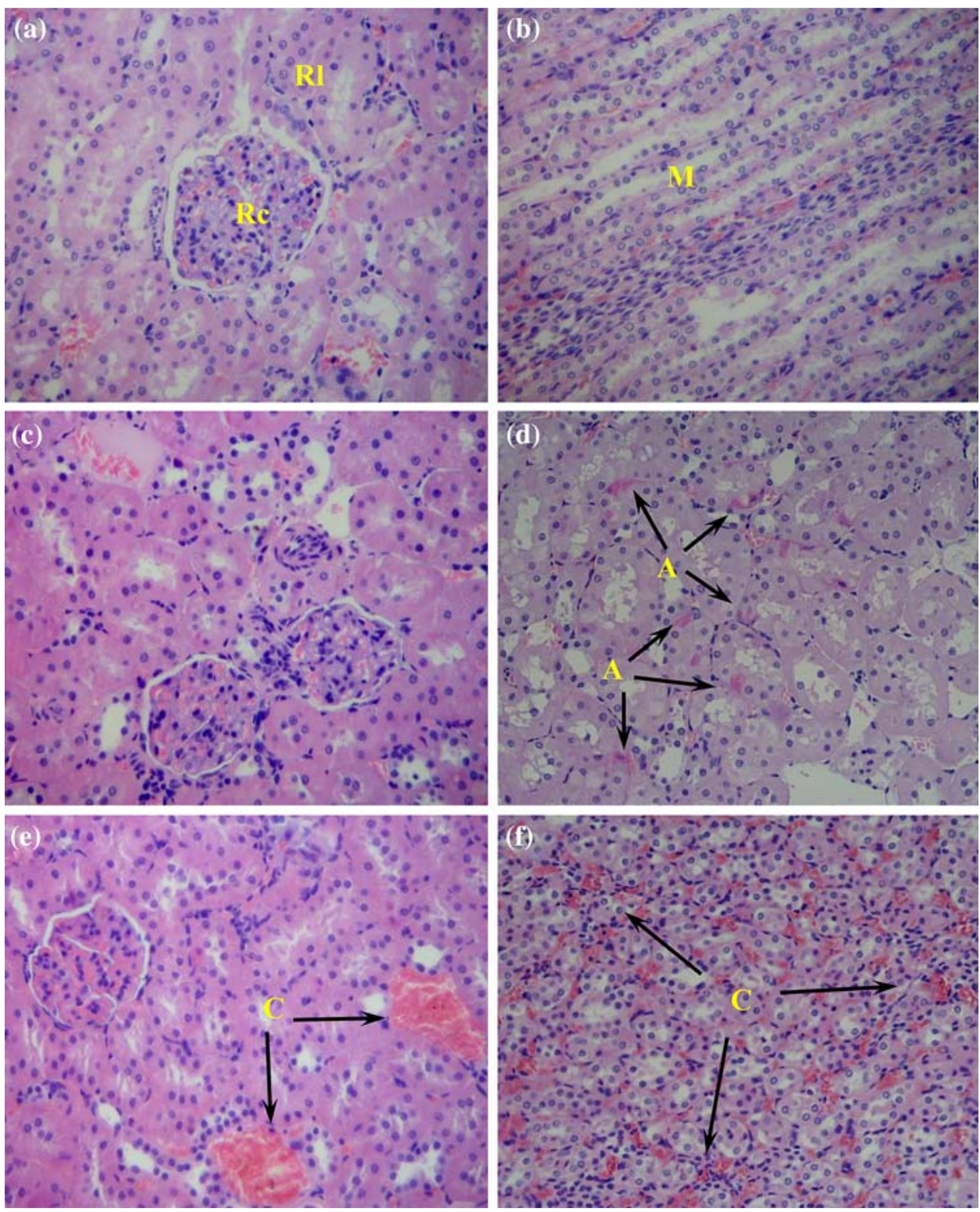

other concomitant symptoms existing in the spleen and brain was observed in PFC-treated groups (Fig. 8a, b), which could contribute to illuminating the immunotoxicity, neurotoxicity, and further reproductive toxicity of PFCs. Contrary, slight splenic atrophy was observed in mice by Yang et al. (2000) and in rats by Goldenthal et al. (1978). No distinct pathological change was found in the testicles herein. Differences in exposure factors such as dose, duration, administration way, and so on, might contribute to the different toxicological effects.

\section{Accumulation and Distribution of PFOA and PFOS in Rats}

Concentrations of PFOA and PFOS in main rat organs and tissues, including whole blood, liver, kidney, lung, heart, spleen, testicle, and brain, were analyzed to elucidate the accumulation and distribution patterns of the tested chemicals and to explain the potential toxicological effects caused in the target organs.

As shown in Table 1, the highest PFOA concentration ( $228 \mu \mathrm{g} / \mathrm{g}$ in G1) existed in the kidney and the liver tissues also contained comparable high levels of PFOA $(218 \mu \mathrm{g} / \mathrm{g}$ in G1 and $196 \mu \mathrm{g} / \mathrm{g}$ in G2), showing that the kidney and the liver might serve as the main target organs for PFOA exposure. Potential toxicological effects were accordingly induced, as confirmed by histopathological alterations described earlier (Figs. 5b, c, and 7c, d). The lung, with obvious histopathological damages shown in Fig. 6b, c, contained relatively high levels of PFOA $(63.0 \mu \mathrm{g} / \mathrm{g}$ in $\mathrm{G} 1$ and $64.3 \mu \mathrm{g} / \mathrm{g}$ in G2), indicating that it could serve as a sensitive organ for PFOA exposure. The 


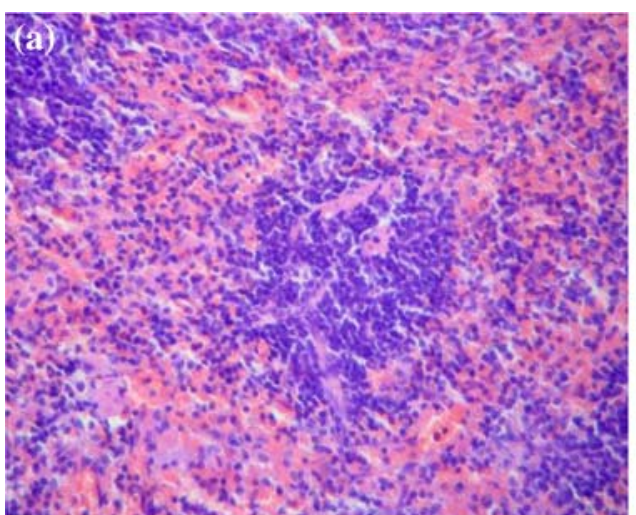

Fig. 8 Histopathological sections of rat spleen and brain from the control and exposure groups. H\&E, $\times 200$. (a) spleen from $20 \mathrm{mg} /$ $\mathrm{kg} /$ day of PFOA; (b) brain from $20 \mathrm{mg} / \mathrm{kg} / \mathrm{day}$ of PFOS. No gross pathological changes were observed in the spleen and brain from the control, they have a normal and clear configuration (images not shown). Congestion (C) accompanied by slight dilatation in splenic antrum was found in the $20-\mathrm{mg} / \mathrm{kg} / \mathrm{day}$ exposure groups. Main

PFOA accumulation levels in the other tested tissues were in the order of (heart, whole blood) $>$ testicle $>$ (spleen, brain). A 28-day exposure test by Ylinen et al. (1990) showed the highest PFOA level was found in serum, which indicated the key role of blood in carrying PFOA around the body after oral administration of this compound. Based on the results in Table 1, it was obvious that similar levels existed in various tissues exposed to both PFOA doses. It has been suggested that PFOA could bind with some specific proteins, such as albumin (an important binding protein) in serum or tissues (Han et al. 2005). Once the binding sites of PFOA in serum or tissues came to saturation, the excretion of PFOA would increase in

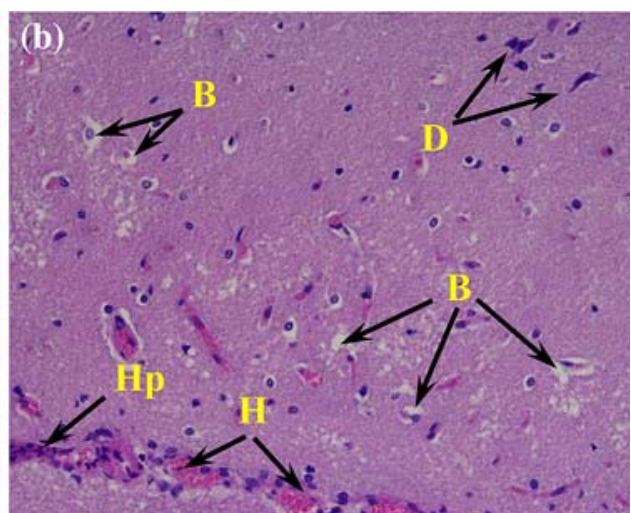

histopathological alterations found in exposure groups were focal hyperplasia (Hp) of cerebral gliocytes, dilatation and congestion in inferior caval veins of cerebral arachnoid mater accompanied by slight focal hemorrhage $(\mathrm{H})$, broadened gap (B) around veins and neurocytes, and focal demyelination (D) of nerve fibers, which were especially discerned in the $20-\mathrm{mg} / \mathrm{kg} /$ day exposure groups

urine and feces (Han et al. 2003). It was thus reasonable that no dose-related accumulation was found in PFOA exposure herein.

According to the analytical results, the PFOS accumulation levels came in the order of liver $>$ heart $>$ kidney $(>$ whole blood $)>$ lung $>$ (testicle, spleen, brain). The liver, as the predominant accumulation tissue for PFOS $(648 \mu \mathrm{g} / \mathrm{g}$ in G4), showed corresponding histological changes as shown in Fig. 5c, d. There is evidence that some specific proteins in the liver can bind with PFOS, which might contribute to the preferential accumulation of PFOS therein (Luebker et al. 2002). As a sensitive organ, the lung was reported to be one of the important targets based on

Table 1 Concentrations of PFOA and PFOS in rat whole blood $(\mu \mathrm{g} / \mathrm{mL})$ and various tissues $(\mu \mathrm{g} / \mathrm{g})$ after 28 days of exposure

\begin{tabular}{|c|c|c|c|c|c|}
\hline \multirow[t]{3}{*}{ Tissues } & $\mathrm{PFOA} / \mathrm{PFOS}^{\mathrm{a}}$ & \multicolumn{2}{|c|}{ Concentration of PFOA } & \multicolumn{2}{|c|}{ Concentration of PFOS } \\
\hline & \multicolumn{5}{|c|}{ Daily exposure dose by gavage } \\
\hline & 0 (Control) & $5 \mathrm{mg} / \mathrm{kg} / \mathrm{day}$ PFOA & $20 \mathrm{mg} / \mathrm{kg} /$ day PFOA & $5 \mathrm{mg} / \mathrm{kg} /$ day PFOS & $20 \mathrm{mg} / \mathrm{kg} /$ day PFOS \\
\hline Blood & $\mathrm{ND}^{\mathrm{b}}$ & $39.2 \pm 14.4$ & $58.8 \pm 17.6$ & $72.0 \pm 25.7$ & $\mathrm{NS}^{\mathrm{c}}$ \\
\hline Liver & ND & $218 \pm 21$ & $196 \pm 10$ & $345 \pm 40$ & $648 \pm 17$ \\
\hline Kidney & ND & $228 \pm 37$ & $209 \pm 74$ & $93.9 \pm 13.6$ & $248 \pm 26$ \\
\hline Lung & ND & $63.0 \pm 11.3$ & $64.3 \pm 15.9$ & $46.6 \pm 17.8$ & $228 \pm 122$ \\
\hline Heart & ND & $35.5 \pm 17.6$ & $34.6 \pm 18.0$ & $168 \pm 17$ & $497 \pm 64$ \\
\hline Spleen & ND & $13.6 \pm 2.4$ & $6.92 \pm 9.31$ & $38.5 \pm 11.8$ & $167 \pm 64$ \\
\hline Testicle & ND & $16.7 \pm 16.9$ & $16.8 \pm 19.2$ & $39.5 \pm 10.0$ & $127 \pm 11$ \\
\hline Brain & ND & $10.5 \pm 9.8$ & $7.20 \pm 6.03$ & $13.6 \pm 1.0$ & $146 \pm 34$ \\
\hline
\end{tabular}

Note: Data are expressed as mean $\pm \operatorname{SD}(n \geq 5)$

${ }^{a}$ The concentrations of PFOA and PFOS in the control group were measured

b Not detected. The data were lower than the detection limits (PFOA: 150 pg/g; PFOS: 110 pg/g)

${ }^{c}$ No sample available because all 10 rats died during the experiment 
acute- and chronic-exposure experiments in rodents (Dean et al. 1978; Grasty et al. 2003), which was in good agreement with the results $(46.6 \mu \mathrm{g} / \mathrm{g}$ in $\mathrm{G} 3$ and $228 \mu \mathrm{g} / \mathrm{g}$ in G4) obtained herein. The heart, playing the key role in the circulatory system, was abundant with blood. It faced more opportunities of PFOS exposure carried by the blood, leading to the high levels (497 $\mu \mathrm{g} / \mathrm{g}$ in G4) therein. The kidney contained relatively high levels of PFOS $(93.9 \mu \mathrm{g} / \mathrm{g}$ in G3 and $248 \mu \mathrm{g} / \mathrm{g}$ in G4), showing that it was important in the metabolism of absorbed PFOS (Kouji et al. 2005). The occurrence of PFOS in the brain indicated that it could cross the blood-brain barrier and result in potential adverse effects on rats, which was confirmed by the finding of the increase of serum corticosterone levels and the decrease of serum leptin concentrations due to PFOS exposure (Austin et al. 2003). Surprisingly, PFOS concentrations in the brains of rats in G4 $(146 \mu \mathrm{g} / \mathrm{g})$ were about 10 -fold higher than those in G3, although the exposure dose of G4 was only fourfold of G3. It might be explained by the fact that the higher-dose administration might cause a significant increase in the permeability of the pollutant to cross the blood-brain barrier, which induced a relatively high accumulation of PFOS in the brain. Dose-related accumulation of PFOS was found in various tissues at the given exposure levels herein, showing the potential high bioaccumulation ability for PFOS in rats. Easy absorption (orally $>95 \%$ ) and poor elimination of PFOS (Lau et al. 2004) might well explain its bioaccumulation profiles.

When the accumulation profiles between PFOA and PFOS are compared, it can be noted that PFOA levels in the kidneys from G1 were much higher than PFOS levels in the corresponding organs from G3 in spite of the same exposure doses used for both compounds. However, in whole blood and the other tested tissues, PFOA levels were relatively lower or at similar levels compared to PFOS. It could be explained that urinary excretion is the primary route for depuration of PFOA in laboratory animals (Butenhoff et al. 2004; Kemper 2003), leading to the preferential deposition of PFOA in the kidney. It has been testified that renal transport of PFOA into urine is by a transporter, whose expression is regulated by sex hormones (Kudo et al. 2002). Transporter proteins might participate in either secretion from blood to the urinary filtrate or in resorption from the latter back into the former. The requirement of urinary clearance for PFOA elevated transporter protein levels in urinary filtrate, which accelerated the resorption of PFOA in the kidney (Andersen et al. 2006). It led to a relatively high accumulation of PFOA therein even at low exposure levels. However, for PFOS, a lower renal clearance was observed, which was confirmed by the relatively longer half-life ( $>90$ days) in the organisms when compared to PFOA (Harada et al. 2005; Thibodeaux et al. 2003).
The available toxicokinetics studies indicated the slower elimination of PFOS compared to PFOA (Johnson et al. 1979; Ohmori et al. 2003), which was confirmed by accumulation profiles of both compounds observed herein (Table 1). Previous research also showed that $\mathrm{LD}_{50}$ values of PFOA and PFOS for male rats were $430-680 \mathrm{mg} / \mathrm{kg}$ and $233 \mathrm{mg} / \mathrm{kg}$, respectively (Dean et al. 1978; Kennedy et al. 2004). The no-observed-adverse effect level (NOAEL) values in rats were $1.5 \mathrm{mg} / \mathrm{kg}$ for PFOA (US EPA 2005) and $0.025 \mathrm{mg} / \mathrm{kg}$ for PFOS (OECD 2002). The occurrence of behavior abnormity (Figs. 1, 2), viscera indexes alterations (Figs. 3, 4), histopathological changes (Figs. 5-8) in the rats treated with PFOA and PFOS definitely indicated that PFOS was more toxic than PFOA.

In conclusion, the toxicological effects induced by PFOA and PFOS on the exposed rats might be helpful in the assessment of environmental risk and the threat to human health posed by universal PFCs pollution.

Acknowledgments This work was jointly supported by the Chinese Academy of Sciences (KZCX2-YW-420-21), the National Natural Science Foundation of China $(20737003,40503014)$, and the National Science and Technology Ministry of China (2006BAK02A06-2).

\section{References}

Abdellatif AG, Preat V (1990) Peroxisome proliferation and modulation of rat liver carcinogenesis by 2, 4-dichlorophenoxyacetic acid, 2, 4, 5-trichlorophenoxyacetic acid, perfluorooctanoic acid and nafenopin. Carcinogenesis 11:1899-1902. doi:10.1093/ carcin/11.11.1899

Abdellatif AG, Preat V (1999) The modulation of rat liver carcinogenesis by perfluorooctanoic acid, a peroxisome proliferator. Toxicol Appl Pharm 111:530-537. doi:10.1016/0041-008X(91) 90257-F

Andersen ME, Clewell HJIII, Tan YM, Butenhoff JL, Olsen GW (2006) Pharmacokinetic modeling of saturable, renal resorption of perfluoroalkylacids in monkeys: probing the determinants of long plasma half-lives. Toxicology 227:156-164. doi:10.1016/ j.tox.2006.08.004

Austin ME, Kasturi BS, Barber M, Kannan K, MohanKumar PS, MohanKumar SMJ (2003) Neuroendocrine effects of perfluorooctane sulfonate in rats. Environ Health Perspect 11:14851489

Batenburg JJ (1992) Surfactant phospholipids: synthesis and storage. Am J Physiol 262:L367-L385

Berthiaume J, Wallace KB (2002) Perfluorooctanoate, perfluorooctane sulfonate and $N$-ethyl-perfluorooctanesulfonamido ethanol: Peroxisome proliferation and mitochondrial biogenenesis. Toxicol Lett 129:23-32. doi:10.1016/S0378-4274(01)00466-0

Butenhoff JL, Kennedy JGL, Hinderliter PM et al (2004) Pharmacokinetics of perfluorooctanoate in Cynomolgus monkeys. Toxicol Sci 82:394-406. doi:10.1093/toxsci/kfh302

Butenhoff JL, York R, Seacat A, Luebker D (2002) Perfluorooctanesulfonate-induced perinatal mortality in rat pups is associated with a steep dose-response. Toxicologist 66:25

Chen LC, Tatum V, Glauert HP, Chow CK (2001) Peroxisome proliferator perfluorodecanoic acid alters glutathione and related enzymes. J Biochem Mol Toxicol 15:107-112. doi:10.1002/jbt.6 
Creuwels LAJM, van Golde LMG, Haagsman HP (1997) The pulmonary surfactant system: biochemical and clinical aspects. Lung 175:1-39. doi:10.1007/PL00007554

Dean WP, Jessup DC, Thompson G, Romig G, Powell D (1978) Fluorad fluorochemical surfactant FC-95 acute oral toxicity $\left(\mathrm{LD}_{50}\right)$ study in rats. Study No.137-083. International Research and Development Corporation, Mattawan, MI

DePierre JW (2002) Effects on rodents of perfluorofatty acids. In: Neilson $\mathrm{AH}$ (ed) The handbook of environmental chemistry. Springer-Verlag, Berlin, pp 203-248

DuPont Haskell Laboratory (1982) Excretion and disposition of 14Cammonium perfluorooctanoate in male and female rats, mice, hamsters, and rabbits. US EPA Public Docket AR-226. US Environmental Protection agency, Washington, DC

Dzhekova SS, Bogdanska J, Stojkova Z (2001) Peroxisome proliferators: their biological and toxicological effects. Clin Chem Lab Med 39:468-474. doi:10.1515/CCLM.2001.076

Fisher AB, Dodia C (2001) Lysosomal-type PLA2 and turnover of alveolar DPPC. Am J Physiol 280:L748-L754

Giesy JP, Kannan K (2001) Global distribution of perfluorooctane sulfonate in wildlife. Environ Sci Technol 35:1339-1342. doi: $10.1021 / \mathrm{es} 001834 \mathrm{k}$

Giesy JP, Kannan K (2002) Perfluorochemical surfactants in the environment. Environ Sci Technol 36:146A-152A

Goldenthal EI, Jessup DC, Geil RG, Mehring JS (1978) Ninety-day subacute rat toxicity study. Study No. 137-085. International Research and development Corporation, Mattawan, MI

Grasty RC, Grey BE, Lau CS, Rogers JM (2003) Prenatal window of susceptibility to perfluorooctane sulfonate-induced neonatal mortality in the Sprague-Dawley rat. Birth Defects Res Part BDev Reprod Toxicol 68:465-471

Guruge KS, Yeung LWY, Yamanaka N et al (2007) Gene expression profiles in rat liver treated with perfluorooctanoic acid (PFOA). Toxicol Sci 89:93-107. doi:10.1093/toxsci/kfj011

Hallman M, Glumoff V, Ramet M (2001) Surfactant in respiratory distress syndrome and lung injury. Comp Biochem Physiol A: Mol Integr Physiol 129:287-294. doi:10.1016/S1095-6433(01) 00324-5

Han X, Kermper RA, Jepson GW (2005) Subcellular distribution and protein binding of perfluorooctanoic acid in rat liver and kidney. Drug Chem Toxicol 28:197-209. doi:10.1081/DCT-200052547

Han X, Snow TA, Kemper RA, Jepson GW (2003) Binding of perfluorooctanoic acid to rat and human plasma proteins. Chem Res Toxicol 16:775-781. doi:10.1021/tx034005w

Hansen KJ, Clemen LA, Ellefson ME, Johnson HO (2001) Compound-specific, quantitative characterization of organic fluorochemicals in biological matrices. Environ Sci Technol 35:766-770. doi:10.1021/es001489z

Harada K, Inoue K, Morikawa A, Yoshinaga T, Saito N, Koizumi A (2005) Renal clearance of perfluorooctane sulfonate and perfluorooctanoate in humans and their species-specific excretion. Environ Res 99:253-261. doi:10.1016/j.envres.2004.12.003

Haughom B, Spydevold Ø (1992) The mechanism underlying the hypolipemic effect of perfluorooctanoic acid (PFOA), perfluorooctane sulphonic acid (PFOSA) and clofibric acid. Biochim Biophys Acta 1128:65-72

Hosokawa M, Satoh T (1993) Differences in the induction of carboxylesterase isozymes in rat iver microsomes by perfluorinated fatty acids. Xenobiotica 23:1125-1133

Houde M, Martin JW, Letcher RJ, Solomon KR, Muir DG (2006) Biological monitoring of polyfluoroalkyl substances: a review. Environ Sci Technol 40:4138-4144. doi:10.1021/es060233b

Hu WY, Jones PD, Celius T, Giesy JP (2005) Identification of genes responsive to PFOS using gene expression profiling. Environ Toxicol Pharmcol 19:57-70. doi:10.1016/j.etap.2004.04.008
Ikeda T, Aiba K, Fukuda K, Tanaka M (1985) The induction of peroxisome proliferation in rat liver by perfluorinated fatty acids, metabolically inert derivatives of fatty acids. J Biochem 98:475482

Johnson JD, Gibson SJ, Ober RE (1979) Extent and route of excretion and tissue distribution of total carbon-14 in rats after a single i.v. dose of FC-95-14C. Project No. 8900310200, Riker Laboratories, Inc., St. Paul, MN [EPA Docket No. 8(e)HQ-1180-00374]

Kannan K, Corsolini S, Falandysz J et al (2004) Perfluorooctanesulfonate and related fluorochemicals in human blood from several countries. Environ Sci Technol 38:4489-4495. doi:10.1021/es0493446

Kemper R (2003) Perfluorooctanoic acid: toxicokinetics in the rat. Project ID: DuPont 7473. US EPA public docket, administrative record AR-226-1499

Kennedy GL, Butenhoff JL, Olsen GW et al (2004) The toxicology of perfluorooctanoate. Crit Rev Toxicol 34:351-384. doi: 10.1080/10408440490464705

Key BD, Howell RD, Criddle CS (1997) Fluorinated organics in the biosphere. Environ Sci Technol 9:2445-2554. doi:10.1021/ es961007c

Kouji H, Kayoko I, Akiko M, Takeo Y, Norimitsu S, Akio K (2005) Renal clearance of perfluorooctane sulfonate and perfluorooctanoate in humans and their species-specific excretion. Environ Res 99:253-261. doi:10.1016/j.envres.2004.12.003

Kudo N, Katakura M, Sato Y, Kawashima Y (2002) Sex hormoneregulated renal transport of perfluorooctanoic acid. Chem Biol Interact 139:301-316. doi:10.1016/S0009-2797(02)00006-6

Lau C, Butenhoff JL, Rogers JM (2004) The developmental toxicity of perfluoroalkyl acids and their derivatives. Toxicol Appl Pharm 198:231-241. doi:10.1016/j.taap.2003.11.031

Lau C, Thibodeaux JR, Hanson RG et al (2003) Exposure to perfluorooctane sulfonate during pregnancy in rat and mouse. II: Postnatal evaluation. Toxicol Sci 74:382-392. doi:10.1093/ toxsci/kfg 122

Luebker DJ, Hansen KJ, Bass NM, Buttenhoff JL, Seacat AM (2002) Interactions of fluorochemicals with rat liver fatty acid-binding protein. Toxicology 176:175-185. doi:10.1016/S0300-483X(02) 00081-1

O’Brien ML, Cunningham ML, Spear BT, Glauert HP (2001) Effects of peroxisome proliferators on glutathione and glutathionerelated enzymes in rats and hamsters. Toxicol Appl Pharmacol 171:27-37. doi:10.1006/taap.2000.9111

OECD (2002) Hazard assessment of perfluorooctanesulfonate (PFOS) and its salts. Unclassified ENV/JM/RD(2002)17/Final. Document No. JT00135607. Organisation for Economic Co-operation and Development, Paris

Ohmori K, Kudo N, Katayama K, Kawashima Y (2003) Comparison of the toxicokinetics between perfluorocarboxylic acids with different carbon chain length. Toxicology 184:135-140. doi: 10.1016/S0300-483X(02)00573-5

Panaretakis T, Shabalina IG, Grandér D, Shoshan MC, DePierre JW (2001) Reactive oxygen species and mitochondria mediate the induction of apoptosis in human hepatoma HepG2 cells by the rodent peroxisome proliferator and hepatocarcinogen, perfluorooctanoic acid. Toxicol Appl Pharm 173:56-64. doi:10.1006/taap. 2001.9159

Pastoor TP, Lee KP, Perri MA, Gillies PJ (1987) Biochemical and morphological studies of ammonium perfluorooctanoate-induced hepatomegaly and peroxisome proliferation. Exp Mol Pathol 47:98-109. doi:10.1016/0014-4800(87)90011-6

Seacat AM, Thomford PJ, Hansen KJ et al (2003) Sub-chronic dietary toxicity of potassium perfluorooctanesulfonate in rats. Toxicology 83:117-131. doi:10.1016/S0300-483X(02)00511-5

Thibodeaux JR, Hanson RG, Rogers JM et al (2003) Exposure to perfluorooctane sulfonate during pregnancy in rat and mouse. I: 
Maternal and prenatal evaluations. Toxicol Sci 74:369-381. doi: 10.1093/toxsci/kfg121

US EPA (2005) Draft risk assessment of the potential human health effects associated with exposure to perfluorooctanoic acid. US Environmental Protection Agency, Washington, DC

Yang Q, Xie Y, Depierre JW (2000) Effects of peroxisome proliferators on the thymus and spleen of mice. Clin Exp Immunol 122:219-226. doi:10.1046/j.1365-2249.2000.01367.x
Ylinen M, Kojo A, Hanhijärvi H, Peura P (1990) Disposition of perfluooctanoic acid in the rat after single and subchronic administration. Bull Environ Contam Toxicol 44:46-53. doi: 10.1007/BF01702360

Zhang P, Shi YL, Cai YQ, Mou SF (2007) Determination of perfluorinated compounds in water samples by high performance liquid chromatography-electrospray tandem mass spectrometry. Chin J Anal Chem 35:969-972 